\title{
Contemporary management of coronary heart disease
}

\author{
IBA Menown \\ Consultant Cardiologist, Craigavon Cardiac Centre, Craigavon, Northern Ireland, and President, Irish Atherosclerosis Society
}

\begin{abstract}
Coronary heart disease remains the leading cause of mortality in the UK. This review focuses on the contemporary management of patients with acute coronary syndromes and those with stable angina, including the role of primary percutaneous coronary intervention versus fibrinolytic therapy in a UK setting, current and emerging antiplatelet and anticoagulant therapies and the latest guidance on secondary prevention/lifestyle modification.
\end{abstract}

KEYWORDS Angina, anti-thrombotic therapy, coronary heart disease, myocardial infarction, percutaneous coronary intervention, secondary prevention
Correspondence to IBA Menown, Craigavon Cardiac Centre, Craigavon Hospital Craigavon BT63 5QQ, Northern Ireland, UK

tel. +44 (0)28 386I 2902

e-mail

ian.menown@southerntrust.hscni.net

DECLARATION OF INTERESTS No conflict of interests declared.

Although important advances in the prevention and treatment of coronary heart disease (CHD) have occurred over the past 15 years, it remains the largest single cause of death in the UK. Clinical presentation may come in many forms and given its high prevalence, particularly in older age groups, a low threshold for suspected diagnosis of CHD is essential. This review focuses on the contemporary management of two broad clinical groupings: those with acute coronary syndromes (ACS) and those with stable angina.

\section{ACUTE CORONARY SYNDROMES}

It is now preferable to classify ACS by the presence or absence of ST elevation on the initial 12-lead electrocardiogram (ECG) as:

I. ST elevation myocardial infarction (STEMI), or

2. non-ST elevation ACS (sub-classified as non-STEMI if cardiac biomarkers are elevated or as unstable angina if there is no biomarker elevation).

An assessment of the initial ECG guides appropriate acute therapy (as opposed to the previous classification based on the presence or absence of $Q$ waves on the discharge ECG).

\section{STEMI}

The primary goal of acute therapy in STEMI is establishing reperfusion in the culprit artery. This may be attempted by primary percutaneous coronary intervention $(\mathrm{PCl})$ (Figure I) or fibrinolytic therapy. Although primary $\mathrm{PCl}$ is potentially more effective, in most clinical scenarios it incurs an additional ('PCl-related') delay compared with immediate fibrinolytic therapy. The acceptable limit for $\mathrm{PCl}$-related delay, beyond which outcomes may be less favourable than with immediate fibrinolytic therapy, is typically around $110-120$ minutes, although this is influenced by time from pain onset, infarct location and patient age. The 2008 European STEMI guideline recommends $\mathrm{PCl}$ as the preferred treatment strategy where feasible (class la recommendation), provided the expected time from first medical contact to balloon/ device is $<120$ minutes (and $<90$ minutes for those presenting early with large infarcts but low bleeding risk). Primary $\mathrm{PCl}$ is also indicated, irrespective of the time delay, for patients in shock or with contraindications to fibrinolytic therapy.

Otherwise, in the absence of contraindications, fibrinolytic therapy is the recommended reperfusion method, given ideally pre-hospital, as a bolus fibrinspecific agent. Even with a fully developed UK primary $\mathrm{PCl}$ service, it is estimated that at least $20 \%$ of patients may be better treated initially by fibrinolytic therapy. Following fibrinolytic therapy, serial ECG analysis is essential to identify the $\sim 25 \%$ of patients who at 90 minutes have failed to show $\geq 50 \%$ resolution of ST elevation (as measured at the J point) and thus need to be considered for 'rescue' PCl. Interestingly, an immediate pre-hospital fibrinolytic plus consistent rescue $\mathrm{PCl}$ strategy has been shown to yield similar outcomes to a primary $\mathrm{PCl}$ strategy in the recent FAST-MI registry. Even in patients with successful ECG resolution after fibrinolytic therapy, urgent cardiac catheterisation +/revascularisation is recommended after three but within 24 hours.

\section{Adjunct antithrombotic therapy in STEMI}

Patients undergoing primary $\mathrm{PCl}$ require rapid loading with antiplatelet therapy and intravenous/arterial anticoagulation.

Currently, oral antiplatelet loading is typically undertaken with aspirin (non-enteric coated) $300 \mathrm{mg}$ and clopidogrel 

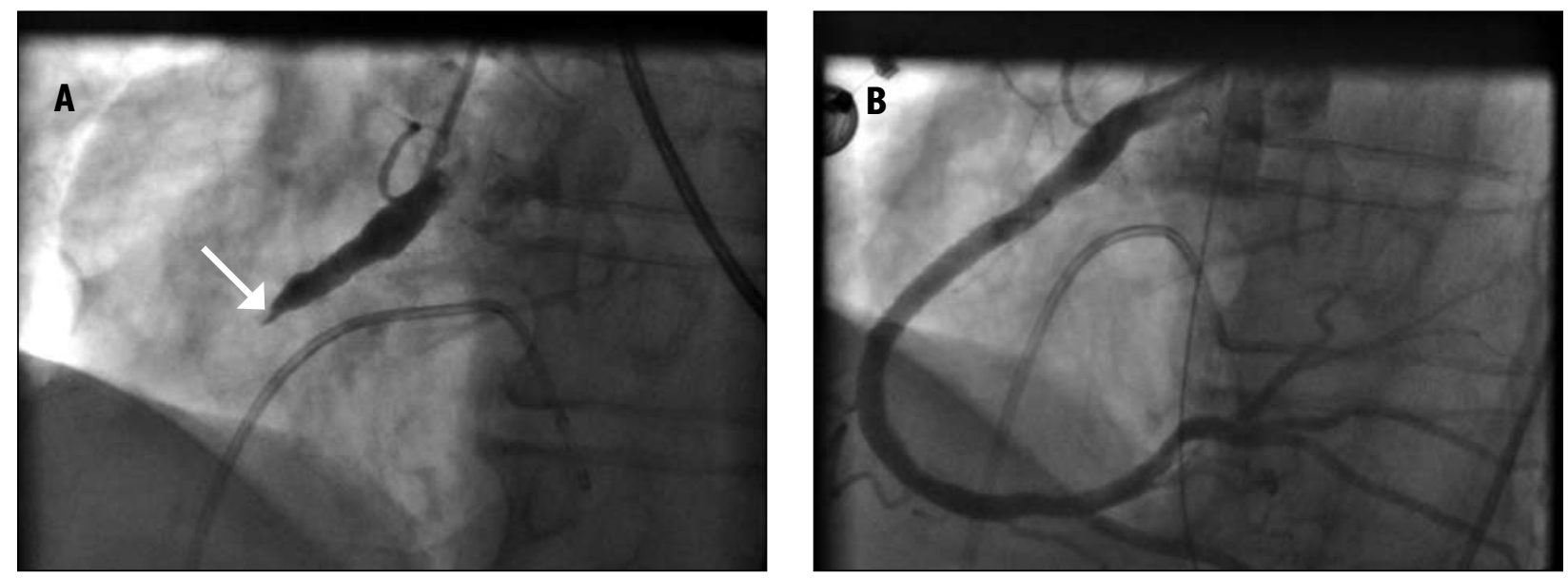

FIGURE I (a) Acutely occluded dominant right coronary artery (arrow) in a patient presenting with extensive inferolateral ST elevation and cardiogenic shock. An intra-aortic balloon pump and temporary pacing wire are in situ. (b) Right coronary artery following successful primary percutaneous coronary intervention.

$600 \mathrm{mg}$ (which achieves acceptable platelet inhibition at $\sim 2$ hours, compared with $\sim 6$ hours following the conventional $300 \mathrm{mg}$ loading dose). The recently approved P2Y 2 receptor blocker prasugrel, given as a $60 \mathrm{mg}$ loading dose, followed by $10 \mathrm{mg}$ once daily, shows faster onset of action and greater platelet inhibition than clopidogrel and appears preferable in the primary $\mathrm{PCl}$ setting (TRITON-TIMI 38). Ticagrelor, a new, nonthienopyridine reversible $\mathrm{P} 2 \mathrm{Y} 12$ receptor blocker which is not yet licensed, also shows faster onset and greater platelet inhibition than clopidogrel, and may be superior in primary $\mathrm{PCl}$ (see Further reading:Wallentin $\mathrm{L}$ et al.).

Intravenous antiplatelet therapy remains indicated in the presence of conventional anticoagulation, that is unfractionated heparin (UFH) or low molecular weight heparin (LMWH). The HORIZONS trial showed that intravenous (IV) direct thrombin inhibition was preferable to the conventional combination of UFH plus glycoprotein $\mathrm{Ilb} /$ IIla blocker with respect to 30-day net clinical adverse events (including a reduction in 30-day cardiac death). The benefit was driven largely by reduced major bleeding. Fondaparinux, the new factor Xa blocker, has been associated with an excess of catheter thrombosis (thought to be due to the absence of anti-lla activity +/lower levels of anti-Xa activity) and is thus contraindicated as the sole anticoagulant during $\mathrm{PCl}$, with additional unfractionated heparin being recommended.

At present, patients receiving fibrinolytic therapy are given adjunct antiplatelet therapy with aspirin loading (300 mg) and clopidogrel (300 mg if $\leq 75$ years; $75 \mathrm{mg}$ if $>75$ years).

While UFH was conventionally used for adjunct anticoagulation to reduce the risk of reocclusion following successful fibrinolysis, the ExTRACT-TIMI 25 trial established the LMWH enoxaparin as the anticoagulant of choice given its significant improvement in efficacy and net clinical benefit compared with UFH. If using enoxaparin, the use of the trial dosing regimen is strongly recommended, that is patients $\leq 75$ years: $30 \mathrm{mg}$ IV bolus, then I $\mathrm{mg} / \mathrm{kg} / \mathrm{I} 2 \mathrm{~h}$ (maximum $100 \mathrm{mg}$ ) subcutaneously; patients $>75$ years: no initial IV bolus, subcutaneous dose $0.75 \mathrm{mg} / \mathrm{kg}$ (maximum $75 \mathrm{mg}$ ); patients with creatinine clearance $<30 \mathrm{ml} / \mathrm{min}$ (dose reduced to I $\mathrm{mg} / \mathrm{kg} / 24 \mathrm{~h}$ ). In contrast, bivalirudin and fondaparinux have not shown any clear benefit over UFH as adjuncts to fibrinolytic therapy.

\section{NON-ST ELEVATION ACS}

Most patients with non-STEMI, and those with unstable angina plus 'high risk' clinical or ECG features (ideally quantified by GRACE or TIMI risk scores), benefit from early (within 72 hours) invasive evaluation +/revascularisation.

At present, acute oral antiplatelet loading with aspirin $300 \mathrm{mg}$ and clopidogrel $300 \mathrm{mg}$ once daily is usually undertaken. Recent and ongoing trials with new alternative P2Y 12 receptor blockers, as discussed above, show superior efficacy to clopidogrel although with increased bleeding risk. With the advent of dual oral antiplatelet therapy, intravenous glycoprotein Ilb/IIla receptor blockade is now usually only given during urgent $\mathrm{PCl}$ but is less commonly required for 'upstream' coronary care unit use.

Unfractionated heparin has largely been replaced as an anticoagulant by one of the newer agents, most commonly the LWMH enoxaparin, which has been associated with reduced death/myocardial infarction (MI) (provided inappropriate 'stacking' with top-up UFH was not undertaken at the time of urgent $\mathrm{PCl}$ as occurred in the SYNERGY trial). If already established on enoxaparin (two or more full subcutaneous doses) and undergoing $\mathrm{PCl}$, the recommended strategy is that no further 
anticoagulant is given if $<8$ hours after the last enoxaparin dose, $0.3 \mathrm{mg} / \mathrm{kg}$ enoxaparin intra-arterial top-up is given if 8-12 hours, and $0.5 \mathrm{mg} / \mathrm{kg}$ enoxaparin intra-arterial bolus is given if $>12$ hours.

Bivalirudin was compared to a heparin (UFH or LMWH) plus glycoprotein Ilb/llla blocker strategy in those undergoing urgent invasive evaluation for non-STE-ACS in the ACUITY study. A 'non-inferior' ischaemic endpoint incidence but less bleeding (hence net clinical advantage favouring bivalirudin) was seen at 30 days, although in those patients who proceeded to $\mathrm{PCl}$ no mortality advantage was seen at one year.

Fondaparinux, compared with enoxaparin in the OASIS5 trial, showed a non-inferior incidence of the primary endpoint (death/Ml/ischaemia) at day nine but less bleeding. Of note, the bleeding benefit was mainly seen in those $>65$ years, enoxaparin was not age-adjusted and full-dose top-up UFH was given relatively early (at/after six hours) to $55 \%$ of enoxaparin vs $20 \%$ of fondaparinux patients undergoing $\mathrm{PCl}$. Catheter thrombosis was more common in fondaparinux patients (well reduced after a protocol amendment recommending top-up UFH). Nevertheless, fondaparinux may have a useful role in more elderly patients with non-STE-ACS not scheduled for urgent invasive evaluation.

\section{SECONDARY PREVENTION AFTER ACS}

Following the stabilisation of ACS, the management of risk factors and establishing evidence-based doses of secondary prevention drugs are essential to optimise early and late outcomes.

\section{General measures (see Further reading: Joint British} Societies' guidelines)

For those who stop smoking, mortality in subsequent years is less than half that of those who continue to smoke. Smoking cessation is thus potentially the most effective of all secondary prevention measures. Cardiac rehabilitation and moderate exercise (to the point of slight breathlessness) for 20-30 minutes on most days is associated with a significant improvement in outcome.

Patients should adopt a Mediterranean-type diet, eat regular fruit and vegetables, and fatty fish at least twice a week. Dietary supplementation for up to four years with fish oil omega-3 fatty acids ( I g daily) may be considered if a patient's general intake of omega- 3 fatty acids is poor ( $<7 \mathrm{~g}$ per week), but other supplements (including beta-carotene and vitamin $\mathrm{C}$ or $\mathrm{E}$ ) are not recommended. Total fat intake should be limited to $\leq 30 \%$ of total energy intake, saturated fats to $\leq 10 \%$ of total fat intake (replacing saturated fats with monounsaturated fats where possible) and dietary cholesterol intake to $\leq 300 \mathrm{mg}$ per day. Weight reduction in overweight patients is encouraged, aiming for an ideal body weight (body mass index $20-25 \mathrm{~kg} / \mathrm{m}^{2}$ ) and avoiding central obesity.

Alcohol intake should be limited to $<2$ I units/week $(<4$ units on any single day) for men and $<14$ units/week $(<3$ units on any single day) for women. Salt intake should be limited to <6 g per day.

\section{Aspirin}

Aspirin irreversibly inhibits cyclo-oxygenase (COX)-I in platelets, thereby limiting the formation of thromboxane $\mathrm{A} 2$ and reducing platelet aggregation. The Antiplatelet Trialists' Collaboration showed a $25 \%$ reduction in death and $\mathrm{MI}$ in post-infarction patients. In both general and post-PCl patients, no benefit has been demonstrated for high dose (i.e. $\geq 300 \mathrm{mg}$ ) aspirin. The conventional dose in the UK is $75 \mathrm{mg}$ lifelong.

\section{Clopidogrel/P2YI 2 blockade}

Adenosine diphosphate (ADP) pathway-induced platelet activation may be blocked by specific inhibition of the P2YI2 ADP receptor. NICE guidelines recommend clopidogrel in addition to aspirin for up to one year after non-STEMI, based on CURE trial data, but only for 30 days after STEMI (since this was the treatment duration in CLARITY). However, the European Society of Cardiology STEMI guideline recommends clopidogrel for one year after STEMI. Maintenance dosage is usually $75 \mathrm{mg}$ once daily, although pre-discharge platelet inhibition assessment may guide higher doses, e.g. 150 $\mathrm{mg}$ once daily in the future. The GRAVITAS trial is under way to assess this strategy.

\section{Beta blockers}

Consensus opinion recommends beta blockers indefinitely after MI, with the strongest evidence in patients with left ventricular (LV) dysfunction. Multiple studies suggest that elevated resting heart rate predicts adverse risk, and titrating the dose to achieve a resting heart rate at least $<70$ beats per minute and, if tolerated, $\sim 60$ beats per minute is considered desirable.

\section{Statins/lipid therapy}

Statin therapy is recommended for all patients with coronary artery disease. Early initiation following Ml may be beneficial through mechanisms of plaque stabilisation, anti-inflammatory effects and restoration of endothelial function. The PROVE-IT trial in patients with recent MI showed that use of an intensive statin strategy (atorvastatin $80 \mathrm{mg}$; median on-treatment low-density lipoprotein cholesterol (LDL-C) $1.6 \mathrm{mmol} / \mathrm{l})$ compared with a moderate statin strategy (pravastatin $40 \mathrm{mg}$; median on-treatment LDL-C $2.5 \mathrm{mmol} / \mathrm{l})$ resulted in a $16 \%$ relative reduction at two years in death/Ml/unstable angina/revascularisation or stroke. Low levels of highdensity lipoprotein $(\mathrm{HDL})$ cholesterol $(<1.0 \mathrm{mmol} / \mathrm{l})$ also predict increased future risk. The independent predictive value of elevated triglycerides is less certain. 


\section{ACE inhibitors}

Angiotensin-converting enzyme (ACE) inhibitors reduce remodelling and improve survival in patients with reduced LV systolic dysfunction post MI. Additionally, ramipril and perindopril have shown improved cardiovascular outcome in patients with risk factors for or established atherosclerotic disease, irrespective of LV function and apparently beyond effects on blood pressure. Thus ACE inhibitors are recommended lifelong for all post-MI patients. Doses should be titrated to the relevant study targets (for example, ramipril $10 \mathrm{mg}$ once daily and perindopril $8 \mathrm{mg}$ once daily). Angiotensin receptor blockers are not routinely recommended post $\mathrm{MI}$, but in individuals known to be intolerant to $\mathrm{ACE}$ inhibitors, valsartan is licensed for patients post $\mathrm{MI}$ with heart failure (based on the VALIANT study).

\section{CHRONIC STABLE ANGINA}

Patients with stable angina (that is, angina which is stable in terms of frequency and severity) should also receive secondary prevention as described above, including general measures, anti-platelet monotherapy, beta blocker or alternative rate-lowering agent (for example, the sino-atrial node blocker ivabradine, or rate-limiting calcium channel blockers verapamil/diltiazem), a statin, and, for most patients, an ACE inhibitor. For lower-risk patients (low risk ideally defined by diagnostic catheterisation as per the COURAGE trial) who are not felt to require revascularisation, additional anti-anginal medication may be considered. It should be noted that apart from rate-lowering anti-anginals there is no current evidence that other anti-anginals reduce the risk of death/MI, and thus their purpose is primarily for symptom relief.

Current options include nitrates (which should be given as a long-acting once a day or asymmetric dosing preparation with a nitrate-free period to reduce tolerance), calcium channel blockers (which may also be useful if there is concurrent hypertension), nicorandil (best reserved for third- or fourth-line use due to the potential risk of gastrointestinal or perineum ulceration) and ranolazine (which is thought to work through inhibiting the late sodium current, thereby reducing intracellular sodium, and hence calcium, overload).

Less established non-drug options include enhanced external counterpulsation and spinal cord stimulation, although these are more controversial and not widely used.

\section{KEY POINTS}

- For ST elevation myocardial infarction (STEMI), primary percutaneous coronary intervention $(\mathrm{PCl})$ is preferred, provided time from first medical contact to balloon/device is $<120$ minutes ( $<90$ minutes if early, large infarct, low bleeding risk; irrespective of delay if shock or contraindication to fibrinolytic therapy).

- During primary $\mathrm{PCl}$, rapid antiplatelet and anticoagulant efficacy is required while avoiding excessive bleeding risk. Prasugrel and bivalirudin are emerging as promising strategies.

- Enoxaparin is the preferred adjunct anticoagulant to fibrinolytic therapy for STEMI.

- Avoid stacking or top-ups of unfractionated heparin on top of enoxaparin when transferring patients from the ward to the catheterisation lab.

- Secondary prevention therapy for established coronary disease includes general lifestyle measures/ risk factor management, aspirin $75 \mathrm{mg}$ daily, clopidogrel $75 \mathrm{mg}$ daily for up to one year, beta blocker (target resting heart rate $60-70$ beats per minute), statin (target LDL-C $<2 \mathrm{mmol} / \mathrm{l}$ ) and an ACE inhibitor (ramipril or perindopril). Consider revascularisation in most patients.

\section{FURTHER READING}

- Anderson JL, Adams CD, Antman EM et al. ACC/AHA 2007 Guidelines for the management of patients with unstable angina/ non-ST-elevation myocardial infarction. Circulation 2007; I I6:e I 48304. doi: I0.1 I6I/CIRCULATIONAHA.107.181940

- Bassand JP, Hamm CW, Ardissino D et al. Guidelines for the diagnosis and treatment of non-ST-segment elevation acute coronary syndromes. Eur Heart J 2007; 28: I598-660. doi:I0.1093/ eurheartj/ehm I6I

- Danchin N, Coste P, Ferrières J et al. Comparison of thrombolysis followed by broad use of percutaneous coronary intervention with primary percutaneous coronary intervention for ST-segment elevation acute myocardial infarction: data from the French Registry on Acute ST-Elevation Myocardial Infarction (FAST-MI). Circulation 2008; I 18:268-76. doi: I0.I I 6I/CIRCULATIONAHA. 107.762765

- Fox K, Garcia MA, Ardissino D et al. Guidelines on the management of stable angina pectoris: executive summary: the Task Force on the Management of Stable Angina Pectoris of the European Society of Cardiology. Eur Heart J 2006; 27:|34|-81. doi:I0.1093/eurheartj/ehl00I

JBS 2: Joint British Societies' guidelines on prevention of cardiovascular disease in clinical practice. Heart 2005; 91 (Suppl 5):vI-52. doi: I0.I I36/hrt.2005.079988

- Mehta SR, Cannon CP, Fox KA et al. Routine vs selective invasive strategies in patients with acute coronary syndromes: a collaborative meta-analysis of randomized trials. JAMA 2005; 293:2908-17. doi:10.1001/jama.293.23.2908

- Van deWerf F, Bax J, Betriu A et al. European Society of Cardiology: Clinical Practice Guidelines. Management of acute myocardial infarction in patients presenting with persistent ST-segment elevation. Eur Heart J 2008; 29:2909-45. doi:I0.1093/eurheartj/ ehn4l6

- Wallentin L, Becker RC, Budaj A et al.Ticagrelor versus clopidogrel in patients with acute coronary syndromes. N Engl J Med 2009;36 I:1045-57. doi:I0.I056/NEJMoa0904327 


\section{SELF-ASSESSMENT QUESTIONS}

I. A 64-year-old man, previously well, develops acute onset ischaemic type chest pain. His wife finds him shortly afterwards and calls for help. A paramedic crew are in attendance $\mathbf{4 0}$ minutes after the onset of pain. A I 2-lead electrocardiogram (ECG) taken by the paramedics shows extensive inferior ST elevation. The nearest accident \& emergency department (A\&E) is $\mathbf{2 5}$ minutes' drive away. The nearest acute percutaneous coronary intervention $(\mathrm{PCl})$ centre is $\mathbf{6 5}$ minutes' drive away. According to European guidelines, which one of the following is the most appropriate course of action?

A. Immediately transfer to the acute $\mathrm{PCl}$ centre for possible primary $\mathrm{PCl}$.

B. Immediate transfer to the nearest A\&E for consideration of urgent fibrinolytic therapy.

C. Pre-hospital streptokinase.

D. Pre-hospital bolus fibrinolytic and transfer for immediate cardiac catheterisation.

E. Pre-hospital bolus fibrinolytic and ECG at 90 minutes to guide need or otherwise for rescue $\mathrm{PCl}$.

2. Which one of the following statements is true?

A. The COURAGE trial showed that patients with stable angina can be safely treated medically without the need for further investigation.

B. First-line anti-anginal therapy is ideally a rate-limiting agent, usually a beta blocker.

C. Nitrates are more effective if given twice a day.

D. Ranolazine is a potassium channel blocker.

E. Ivabradine is particularly useful for patients with angina and atrial fibrillation.

3. For those with non-ST elevation acute coronary syndrome (NSTE-ACS), which one of the following statements is true?

A. Patients with NSTE-ACS but without troponin rise are best treated conservatively unless they have refractory symptoms or ECG changes.

B. Patients with NSTE-ACS may carry a similar risk of adverse cardiovascular outcome as patients with STEMI.
C. In UK clinical practice, glycoprotein Ilb/llla blockers are commenced immediately in the coronary care unit for most patients following a diagnosis of NSTE-ACS.

D. Unfractionated heparin remains the preferred anticoagulant for the initial management of NSTE-ACS.

E. Fondaparinux may have a particular advantage over other anticoagulants in younger patients ( $<65$ years of age).

4. In the setting of ST elevation myocardial infarction (STEMI), which one of the following statements is correct?

A. Fondaparinux is the adjunct anticoagulant of choice for patients likely to need urgent invasive evaluation.

B. Clopidogrel loading with $300 \mathrm{mg}$ achieves acceptable platelet inhibition within one hour.

C. Bivalirudin is best combined with a glycoprotein Ilb/llla blocker during primary $\mathrm{PCl}$.

D. Enoxaparin is the anticoagulant of choice as an adjunct to fibrinolytic therapy.

E. If using fibrinolytic therapy, the first dose of clopidogrel should be delayed for 48 hours.

5. In the treatment of established cardiovascular disease, which one of the following statements is false?

A. Smoking cessation is potentially the most effective secondary prevention measure.

B. Following myocardial infarction, aspirin is associated with a $25 \%$ reduction in death or re-infarction.

C. European guidelines recommend stopping clopidogrel one month after ST elevation myocardial infarction.

D. Beta blockers are recommended following myocardial infarction irrespective of anginal symptoms.

E. Following acute coronary syndrome, intensive lipid therapy to achieve low-density lipoprotein levels as low as $1.6 \mathrm{mmol} / \mathrm{l}$ may be more beneficial than a moderate lipid-lowering strategy.

This paper was originally published as part of the Cardiology module in the RCPE Online Continuing Medical Education Programme. Online CME, including the anwers to these questions and further material, is available to Fellows and Members at: https://members.rcpe.ac.uk/cme.php 